\title{
RESEARCH
}

Open Access

\section{Low levels of pyruvate induced by a positive feedback loop protects cholangiocarcinoma cells from apoptosis}

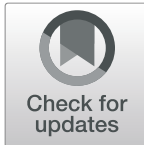

Mingming Zhang ${ }^{1,2,4 \dagger}$, Yida Pan ${ }^{3+}$, Dehua Tang ${ }^{1+}$, Robert Gregory Dorfman ${ }^{5}$, Lei Xu' ${ }^{6}$, Qian Zhou ${ }^{4}$, Lixing Zhou ${ }^{1}$, Yuming Wang ${ }^{1}$, Yang Li ${ }^{6}$, Yuyao Yin ${ }^{1}$, Bo Kong ${ }^{1,7}$, Helmut Friess ${ }^{7}$, Shimin Zhao ${ }^{2,4}$, Jian-lin Wu ${ }^{8^{*}}$, Lei Wang ${ }^{1 *}$ and Xiaoping Zou ${ }^{1 *}$

\begin{abstract}
Background: Cancer cells avidly consume glucose and convert it to lactate, resulting in a low pyruvate level. This phenomenon is known as the Warburg effect, and is important for cell proliferation. Although cMyc has often been described as an oncoprotein that preferentially contributes to the Warburg effect and tumor proliferation, mechanisms of action remain unclear. Histone deacetylase 3 (HDAC3) regulates gene expression by removing acetyl groups from lysine residues, as well as has an oncogenic role in apoptosis and contributes to the proliferation of many cancer cells including cholangiocarcinoma (CCA). HDAC inhibitors display antitumor activity in many cancer cell lines. Cancer cells maintain low levels of pyruvate to prevent inhibition of HDAC but the mechanisms remain elusive. The purpose of our study was to explore the role of cMyc in regulating pyruvate metabolism, as well as to investigate whether the inhibitory effect of pyruvate on HDAC3 could hold promise in the treatment of cancer cells.

Methods: We studied pyruvate levels in CCA cell lines using metabolite analysis, and analyzed the relationship of pyruvate levels and cell proliferation with cell viability analysis. We cultivated CCA cell lines with high or low levels of pyruvate, and then analyzed the protein levels of HDAC3 and apoptotic markers via Western Blotting. We then explored the reasons of low levels of pyruvate by using seahorse analysis and ${ }^{13} \mathrm{C}_{6}$ metabolites tracing analysis, and then confirmed the results using patient tissue protein samples through Western Blotting. Bioinformatics analysis and transfection assay were used to confirm the upstream target of the low levels of pyruvate status in CCA. The regulation of cMyc by HDAC3 was studied through immunoprecipitation and Western Blotting.

(Continued on next page)
\end{abstract}

\footnotetext{
*Correspondence: jlwu@must.edu.mo; 867152094@qq.com;

zouxp@nju.edu.cn

${ }^{+}$Mingming Zhang, Yida Pan and Dehua Tang contributed equally to this work.

${ }^{8}$ State Key Laboratory of Quality Research in Chinese Medicine, Macau Institute for Applied Research in Medicine and Health, Faculty of Chinese Medicine, Macau University of Science and Technology, Avenida Wai Long, Taipa, Macao 442000, People's Republic of China

'Department of Gastroenterology, Nanjing Drum Tower Hospital, the Affiliated Hospital of Nanjing University Medical School, Nanjing University, No.321 Zhongshan Road, 210008 Nanjing, People's Republic of China Full list of author information is available at the end of the article
}

(c) The Author(s). 2019 Open Access This article is distributed under the terms of the Creative Commons Attribution 4.0 International License (http://creativecommons.org/licenses/by/4.0/), which permits unrestricted use, distribution, and reproduction in any medium, provided you give appropriate credit to the original author(s) and the source, provide a link to the Creative Commons license, and indicate if changes were made. The Creative Commons Public Domain Dedication waiver (http://creativecommons.org/publicdomain/zero/1.0/) applies to the data made available in this article, unless otherwise stated. 
(Continued from previous page)

Results: We confirmed downregulated pyruvate levels in CCA, and defined that high pyruvate levels correlated with reduced cell proliferation levels. Downregulated pyruvate levels decreased the inhibition to HDAC3 and consequently protected CCA cells from apoptosis. Synergistically upregulated LDHA, PKM2 levels resulted in low levels of pyruvate, as well as poor patient survival. We also found that low levels of pyruvate contributed to proliferation of CCA cells and confirmed that the upstream target is CMyc. Conversely, high activity of HDAC3 stabilized cMyc protein by preferential deacetylating cMyc at K323 site, which further contributed to the low pyruvate levels. Finally, this creates a positive feedback loop that maintained the low levels of pyruvate and promoted CCA proliferation.

Conclusions: Collectively, our findings identify a role for promoting the low pyruvate levels regulated by c-Myc, and its dynamic acetylation in cancer cell proliferation. These targets, as markers for predicting tumor proliferation in patients undergoing clinical treatments, could pave the way towards personalized therapies.

Keywords: cMyc, Pyruvate, HDAC3, Apoptosis, Cholangiocarcinoma,

\section{Highlights}

We found cMyc decreases pyruvate levels by promoting LDHA and PKM2 levels, this can consequently decrease the inhibition to HDAC3 and protect cancer cells from apoptosis. Conversely, high activity of HDAC3 stabilizes the cMyc protein by preferentially deacetylating cMyc at K323 site, which further contributes to low pyruvate levels. This creates a positive feedback loop that promotes the Warburg effect and cell proliferation of the tumor.

Together, this suggest the low pyruvate levels, regulated by c-Myc and its dynamic acetylation, can serve as a marker for predicting tumor proliferation in patients undergoing clinical treatments. These potential targets could pave the way towards personalized therapies.

\section{Background}

Cancer cells mainly rely on aerobic glycolysis to generate enough energy and intermediates for the malignant behaviors. This so-called Warburg Effect can convert the glycolysis-induced pyruvate into lactate, thus making low pyruvate status in cancer cells [1]. The low pyruvate levels in cells are due to both reduced production and excessive consumption. The reduced pyruvate production comes from the expression of pyruvate kinase, the enzyme responsible for the generation of pyruvate in glycolysis. The splice variant PKM2 (musclespecific pyruvate kinase 2) is expressed specifically in cancer cells in the dimeric form with low catalytic activity, and is predictive of a poor prognosis in CCA patients [2]. The dominant pyruvate consumption comes from the conversion of pyruvate into lactate, and this reaction is mediated by lactate dehydrogenase (LDH). There are different isoforms of tetrameric LDH: LDHA and LDHB, and LDHA is effective in the conversion of pyruvate into lactate. Since tumor cells robustly convert pyruvate into lactate, one would expect a high expression of LDHA in cancer cells as well as cholangiocarcinoma (CCA) [3].
As an oncogene, $c-M y c$ has attracted extensive interest as its potential role for contributing to tumorigenesis. $M Y C$, and $c-M y c$ in particular, is one such oncogene. $M Y C$ was discovered in studies of fulminant chicken tumors caused by oncogenic retroviruses. Subsequently, genomic sequencing efforts identified $c-M y c$ as one of the most highly amplified oncogenes in many different human cancers $[4,5]$. There are various mechanism of MYC-induced tumorigenesis, including increased Warburg effect, and many studies have found that MYC increased metabolic proteins, such as LDH and PKM2 $[6,7]$. Therefore, many studies focus on the therapeutic value of targeting Myc. So far, no small molecules can directly target $\mathrm{c}-\mathrm{Myc}$ in vivo. Both suppressing $\mathrm{c}-\mathrm{Myc}$ transcription by bromodomain inhibitors targeting BRD4 and destabilizing c-Myc protein level by SIRT2 inhibition significantly reduced cancer cell proliferation $[5,8]$. As the stability of c-Myc contributed to tumorigenesis, additional studies have found that the stability of $\mathrm{c}-\mathrm{Myc}$ protein is related to the low acetylation at K323 [9, 10]. The treatment of HDAC inhibitors (HDACi), but not SIRT inhibitors, induced c-Myc K323 acetylation as well as tumorigenesis inhibition, suggesting that at least one of HDACs is the deacetylase of c-Myc [11, 12]. Although cMyc have often been described as preferentially an oncoprotein that contributes to the Warburg effect and tumor proliferation, mechanisms of action still remain unclear.

Genetic or epigenetic alterations, which disrupt proliferation and cell death pathways, are the fundamental event for initiation and progression of cancer [13]. Imbalanced epigenetic networks have been identified in all types of cancers and involve multiple metabolic changes. Unlike genetic mutations, epigenetic modifications are potentially reversible, thereby allowing drugs acting on specific enzymes involved in the epigenetic regulation of gene expression and raising the possibility of epigenetic therapies [12, 14]. Among the epigenetic alterations, 
acetylation has emerged as a key post-translational modification and acetylases have been identified as key metabolic enzymes in cellular regulation [15]. Deacetylases are designated to four classes (I-IV), depending on their amino acid sequence structure [13]. Class I HDACs $(1,2,3$ and 8$)$ play an important role in tumorigenesis and may be candidate targets for many cancer treatments $[16,17]$. Recently, we confirmed high levels of HDAC3 expressed in CCA tissues, and found that this was associated with poor survival in CCA patients. Mechanistically, HDAC3 induced proliferation and protected CCA cells from apoptosis [18]. HDACs inhibitors have been noted for their ability to induce cell cycle arrest and apoptosis of a broad spectrum of cancer cells $[13,16-19]$. Some agents have shown signs of efficacy in clinical trials. SAHA and romidepsin are U.S. Food and Drug Administration (FDA) approved for the treatment of cutaneous T-cell lymphoma [13]. Novel Class I HDACs inhibitors were found to be beneficial for tumorigenesis inhibition as well as the acetylation-induced cMyc degradation [18, 20-22].

Collectively, the mechanisms that underlie cMyc's ability to promote tumorigenesis via the Warburg effect are poorly understood. Thus, the question of whether cMyc acetylation is beneficial for its induced-tumorigenesis still remains unanswered. Here we set out to identify whether low pyruvate levels regulated by c-Myc, and its dynamic acetylation, promote cancer cell proliferation. These targets, as markers of predicting tumor proliferation in patients undergoing clinical treatments, could pave the way towards therapeutic intervention in the treatment of CCA.

\section{Materials and methods}

\section{Ethics, consent and permissions}

All experiments utilizing animal and human samples were approved by the Ethical Committee of Medical Research, Nanjing Drum Tower Hospital, Affiliated Hospital of Nanjing University Medical School.

\section{Cell culture and reagents}

One human intrahepatic biliary epithelial cell line HIBE$\mathrm{piC}$ and six human cholangiocarcinoma (CCA) cell lines HuCCT1, OZ, HuH28, Hccc9810, RBE, and QBC939 were used. HIBEpiC were obtained from the ScienCell (ScienCell, CA, Carlsbad, USA). HuCCT1, OZ, HuH28, and Hccc9810 cells were obtained from the Japanese Collection of Research Bioresources (JCRB) (Tokyo, Japan). RBE cells were obtained from the Institute of Biochemistry and Cell Biology, Shanghai Institutes for Biological Sciences, Chinese Academy of Sciences (Shanghai, China). QBC939 cells were kindly provided by Professor Shuguang Wang from The Third Military Medical University (Chongqing, China). Cells were maintained in Dulbecco's modified Eagle's medium (DMEM) (Invitrogen, Carlsbad, CA, USA) containing $10 \%$ fetal bovine serum (Invitrogen), penicillin (Invitrogen) $(100 \mathrm{U} / \mathrm{ml})$ and streptomycin (Invitrogen) (100 U/ $\mathrm{ml}$ ). For apoptosis experiments, a moderate concentration of $1 \mathrm{mM}$ ethyl pyruvate is present in order to maintain a proper basal apoptotic rate. Full-length HDAC1-3, PKM2, LDHA (wildtype) and cMYC (wildtype and K323R) plasmids were kindly provided by the Zhao lab of Fudan University (Shanghai, China). Cells were transfected with Lipofectamine 3000 (Invitrogen) according to the manufacturer's protocol. The HDAC3 siRNA was commercially purchased from RiboBio (Guangzhou, China), siRNA-HDAC3-1: CCATGACAA TGACAAGGAA, siRNA-HDAC3-2: GCATTGATGAC CAGAGTTA, siRNA-HDAC3-3: GAATATGTCAAGA GCTTCA. HDAC3 shRNA (h) lentiviral particles were commercially purchased from Santa Cruz Biotechnology. RGFP966 (MCE, Monmouth Junction, NJ, USA) was commercially purchased.

\section{Cell viability assay}

Cell viability was determined using the CCK- 8 colorimetric assay in 96-well plates $\left(2 \times 10^{3}\right.$ cells/well $)$ (Dojindo, Minato-ku, Tokyo, Japan) and cultured at $37^{\circ}$ $\mathrm{C}$ with $5 \% \mathrm{CO} 2$. After treatment at the indicated times, $10 \mathrm{ul}$ of CCK- 8 solutions was added to each well. Then, cells were incubated for one and a half hours. The absorbance of the samples at $450 \mathrm{~nm}$ was recorded using a scanning multi-well spectrophotometer. Relative cell viability $(\%)=($ absorbance $450 \mathrm{~nm}$ of treated group - absorbance $450 \mathrm{~nm}$ of blank)/(absorbance $450 \mathrm{~nm}$ of control group - absorbance $450 \mathrm{~nm}$ of blank) $\times 100$.

\section{Cellular pyruvate levels assay}

Cellular pyruvate levels were detected by using Pyruvate Colorimetric/Fluorometric Assay Kit (K609-100, Biovision, Milpitas, California, USA) following the manufacturer's instructions.

\section{Western blot}

Cells were lysed with $0.5 \%$ NP40 lysis buffer and proteins were blotted following standard protocol; except for the detection of acetylation, which used $50 \mathrm{mM}$ Tris $(\mathrm{pH} 7.5)$ with $10 \%(v / \mathrm{v})$ Tween 20 and $1 \%$ peptone (AMRESCO, Solon, OH, USA) as a blocking buffer. Primary and secondary antibodies were diluted in 50 $\mathrm{mM}$ Tris (pH 7.5) with $0.1 \%$ peptone. Signals were probed using the chemiluminescence ECL plus reagent (Thermo, Grand Island, NY, USA) and detected using a Typhoon FLA9500 scanner (GE, Fairfield, CT, USA). Primary antibodies were as follows: HDAC1 (Abcam, Cambridge, UK), HDAC2 (Abcam), HDAC3 (Abcam), cleaved caspase-3 (CST, Danvers, MA, USA), cleaved 
PARP (CST), PARP (CST), PKM2 (Abcam), LDHA (Abcam), cMYC (Abcam), $\beta$-actin (Sigma), FLAG (Abcam), and HA (provided by the Zhao lab of Fudan University).

\section{Deacetylation assay}

Cells were lysed in NP-40 buffer containing $50 \mathrm{mM}$ Tris-HCl (pH 7.5) (Sigma, St Louis, MO, USA), $150 \mathrm{mM}$ $\mathrm{NaCl}$ (Sangon, Shanghai, China), 0.5\% Nonidet P-40 (Sigma), $1 \mu \mathrm{g} / \mathrm{ml}$ aprotinin (Sigma), $1 \mu \mathrm{g} / \mathrm{ml}$ leupeptin (Sigma), $1 \mu \mathrm{g} / \mathrm{ml}$ pepstatin (Sigma), $1 \mathrm{mM}$ Na3VO4 (Sigma) and $1 \mathrm{mM}$ PMSF (Sigma). For immunoprecipitation, $500 \mu \mathrm{l}$ of cell lysate was incubated with HA antibody (provided by the Zhao lab of Fudan University) for $3 \mathrm{~h}$ at $4{ }^{\circ} \mathrm{C}$ with rotation. Then, $30 \mu \mathrm{l}$ of Protein A Agarose (Millipore, Billerica, MA, USA) was added for $12 \mathrm{~h}$ at $4{ }^{\circ} \mathrm{C}$ with rotation, and the beads were washed three times with lysis buffer before proteins were dissolved in loading buffer. Deacetylation assays were carried out in the presence of $5 \mu \mathrm{g}$ enzyme and $0.3 \mu \mathrm{g}$ peptide in $30 \mu \mathrm{l}$ reaction buffer [30 mM HEPES (Sigma), $0.6 \mathrm{mM} \mathrm{MgCl} 2$ (Sangon), $1 \mathrm{mM}$ DTT (Sigma), $1 \mathrm{mM}$ NAD+ (Sigma), 10 mM PMSF (Sigma)]. The deacetylation reaction was incubated for $2 \mathrm{~h}$ at $37^{\circ} \mathrm{C}$ before the mixture was desalted by passing it through a C18 ZipTip (Millipore). The desalted samples were analyzed using a MALDI-TOF/ TOF mass spectrometer (Applied Biosystems, Grand Island, NY, USA). The acetylated peptide used in the assay was TRKDYPAAK (Ac) RVKLDSVR (Glssale, Shanghai, China).

\section{Mitochondrial oxidative phosphorylation analysis}

Oxygen consumption rate (OCR) was detected in real-time with the XF96 Extracellular Flux Analyzer from Seahorse Bioscience, Inc. (North Billerica, MA, USA) following the manufacturer's instructions.

\footnotetext{
Metabolite analysis

${ }^{13} \mathrm{C}$ metabolism labeling experiments have previously been documented [23]. Metabolite extracts were collected and $2 \mathrm{ul}$ of metabolite extract samples were injected for the GC-MS analysis using an Agilent 6980 GC coupled to an Agilent 5973 MS system. Relative metabolite abundances were determined by normalizing abundances of each metabolite to the internal standard and to cell number.
}

\section{DEGs of paired-CCA from TCGA data}

The CCA RNA-Seq data were downloaded from the TCGA database using The GDC Data Portal (https:// portal.gdc.cancer.gov). The mRNA expression data included a total of 18 samples consisting of 9 normal sample and 9 paired-CCA samples. The sequencing data were all publicly available and no ethical issues were involved. The edgeR package in Bioconductor was used to screen the DEGs in CCA and normal liver tissue samples. The edgeR package is based on the negative binomial (NB) distribution, which can correct the overdispersion problem in RNA-seq data by using a Poisson model and a Bayes procedure. Data with expression values of zero were removed. The genes were deemed to be DEGs if $\mid$ FoldChange $\mid>2$, respectively, both with $p$-value $<0.01$ and false discovery rate $(\mathrm{FDR})<0.05$.

\section{Functional annotation}

The Database for Annotation Visualization and Integrated Discovery (DAVID) online tool (https://david.nc ifcrf.gov/) was used to conduct the functional and pathway enrichment analyses in our study. We performed GO and KEGG pathway enrichment analyses to detect the potential biological functions and pathways of the High and low expression genes in CCA.

\section{Immunohistochemistry}

Tumor specimens from human and mice were fixed in $4 \%$ formalin and embedded in paraffin. Two to five human CCA tumor specimens from one patient were used for the IHC study. Standard procedures were followed for IHC except for the detection of targets. The staining intensity for each tissue was calculated by multiplication and the range of this calculation [24].

\section{Ubiquitination assay}

Thirty-six hours following transfection, cells were lysed in 1\% SDS buffer (Tris (Sigma), $0.5 \mathrm{mM}$ EDTA (Sigma) and $1 \mathrm{mM}$ DTT (Sigma), $\mathrm{pH}=7.5$ ), as well as boiled for $10 \mathrm{~min}$. For immunoprecipitation, the lysates were diluted 10-fold in Tris- $\mathrm{HCl}$ buffer. Analyses of ubiquitination were performed using anti-HA blotting.

\section{CCA xenograft model}

Nude mice were purchased from the Department of Laboratory Animal Science, Nanjing Drum Tower Hospital. HuCCT1 cells $\left(5 \times 10^{6}\right)$ in FBS-free medium were subcutaneously injected into the abdomen of mice. The Animal Welfare Committee of Nanjing Drum Tower Hospital approved all procedures involving animals.

\section{Statistics}

Data was expressed as means \pm standard error of the mean (SE). The data was analyzed through one-way ANOVAs followed by post hoc Duncan tests (SPSS 17.0). $P<0.05$ was considered significant. 


\section{Results}

\section{High levels of intracellular pyruvate inhibit the} proliferation of CCA cells

Previous studies have identified pyruvate as an HDAC inhibitor and indicate that tumor cells would silence $\mathrm{Na}+$ -coupled pyruvate transport SLC5A8, as well as convert pyruvate into lactate, as complementary mechanisms to avoid pyruvate-induced cell death [25]. Therefore, we examined the inhibitory effect of pyruvate on CCA. CCK8 assay results showed that marginal growth was inhibitory of pyruvate, even at levels as high as $2 \mathrm{mM}$ in HuCCT1 cells and RBE cells (Fig. 1a \& b). However, ethyl pyruvate, a stable lipophilic derivative of pyruvate which could penetrate the cell membranes directly, could significantly inhibit the proliferation of CCA (Fig. 1a \& b). Consistent with the CCK8 assays, pyruvate levels in HCC cells were significantly increased only after ethyl pyruvate treatment (Fig. 1c). Our previous study confirmed that the highly-expressed HDAC3 was correlated with poor prognosis, and that HDAC3 inhibition is a promising anti-proliferation approach in CCA [18]. To further evaluate the relationship between intracellular pyruvate and CCA proliferation, we screened a normal bile duct epithelial cell line (HIBEpiC) and five human CCA cell lines (QBC939, HuCCT1, Hccc9810, OZ, RBE and HuH28). We found that CCA cells simultaneously have high levels of HDAC3 expression with low levels of SLC5A8 (Fig. 1d). Interestingly, we found that high levels of intracellular pyruvate negatively correlated with cell proliferation (Fig. 1e). Moreover, the low pyruvate levels in cells were further confirmed in fresh cholangiocarcinoma tissues (Fig. 1f).
Collectively, these results reveal that high levels of intracellular pyruvate in CCA cells inhibits its proliferation.

\section{Pyruvate decreases the proliferation of CCA cells by inhibiting HDAC3}

Our previous data screened Class I HDACs and confirmed that only HDAC3 could rescue apoptosis in CCA cell lines [18]. Moreover, Class I HDACs shared a high level of homology with each other (HDAC 2 shares 52\% identity with HDAC3) [26, 27]; thus, pyruvate possibly has a weak inhibitory effect on HDAC1 and 2 as well. To elucidate the direct target of pyruvate, we used an in vitro deacetylation system (Fig. 2a \& b). Pyruvate treatment inhibited HDAC1 and 3 deacetylation activity, but only had a marginal inhibitory effect on HDAC2 (Fig. 2c). To determine if the anti-proliferative effect of ethyl pyruvate in CCA cells was due to HDAC3 inhibition, HDAC3-knockdown CCA cells were treated with ethyl pyruvate. HDAC3 protein levels were unchanged by ethyl pyruvate treatment, suggesting that ethyl pyruvate inhibits the activity of HDAC3 directly (Fig. 2d). Furthermore, ethyl pyruvate treatment significantly promoted cell apoptosis, whereas HDAC3-knockdown counterparts did not show significant apoptosis (Fig. 2d). These results demonstrate that HDAC3 is the main target of pyruvate in inducing CCA cell apoptosis.

\section{Changes in metabolic enzymes contribute to low levels of pyruvate}

One of the guaranteed anticancer strategies is to increase intracellular pyruvate levels [28], so we decided to
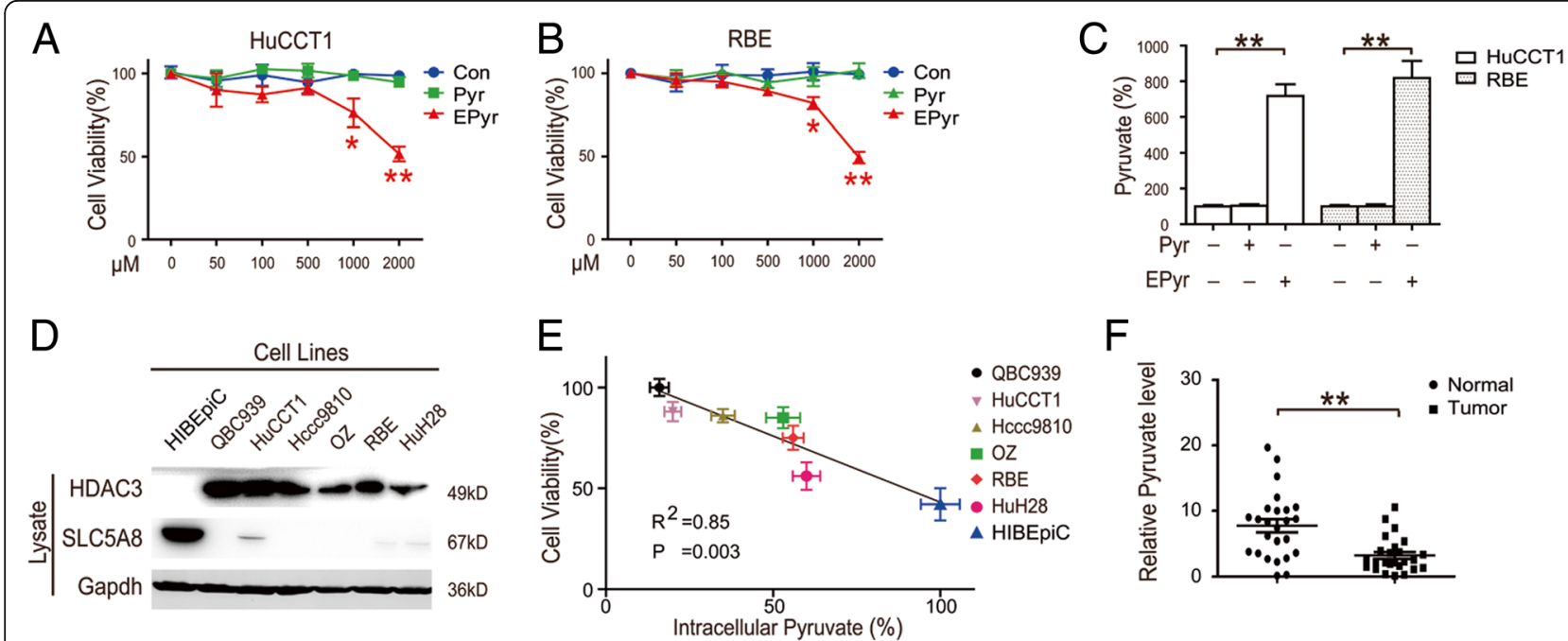

Fig. 1 High levels of intracellular pyruvate in CCA cells inhibits its proliferation. $\mathbf{a}$ and $\mathbf{b}$ CCA cells were treated with pyruvate and ethyl pyruvate for $48 \mathrm{~h}$, then quantified via CCK-8 assay. c CCA cells were treated as indicated for $48 \mathrm{~h}$; the intracellular pyruvate was quantified and normalized to its protein level. $\mathbf{d}$ HDAC3 and SLC5A8 protein levels were detected by western blot. e The correlation between the cell viability and its intracellular pyruvate level in CCA cells. e Pyruvate levels in fresh tumor and adjacent normal tissues from 25 CCA patients were analyzed and normalized to its protein level. Data represent the Mean \pm SEM, $n \geq 3 .{ }^{*} p<0.05,{ }^{* *} p<0.01$, NS not significant 


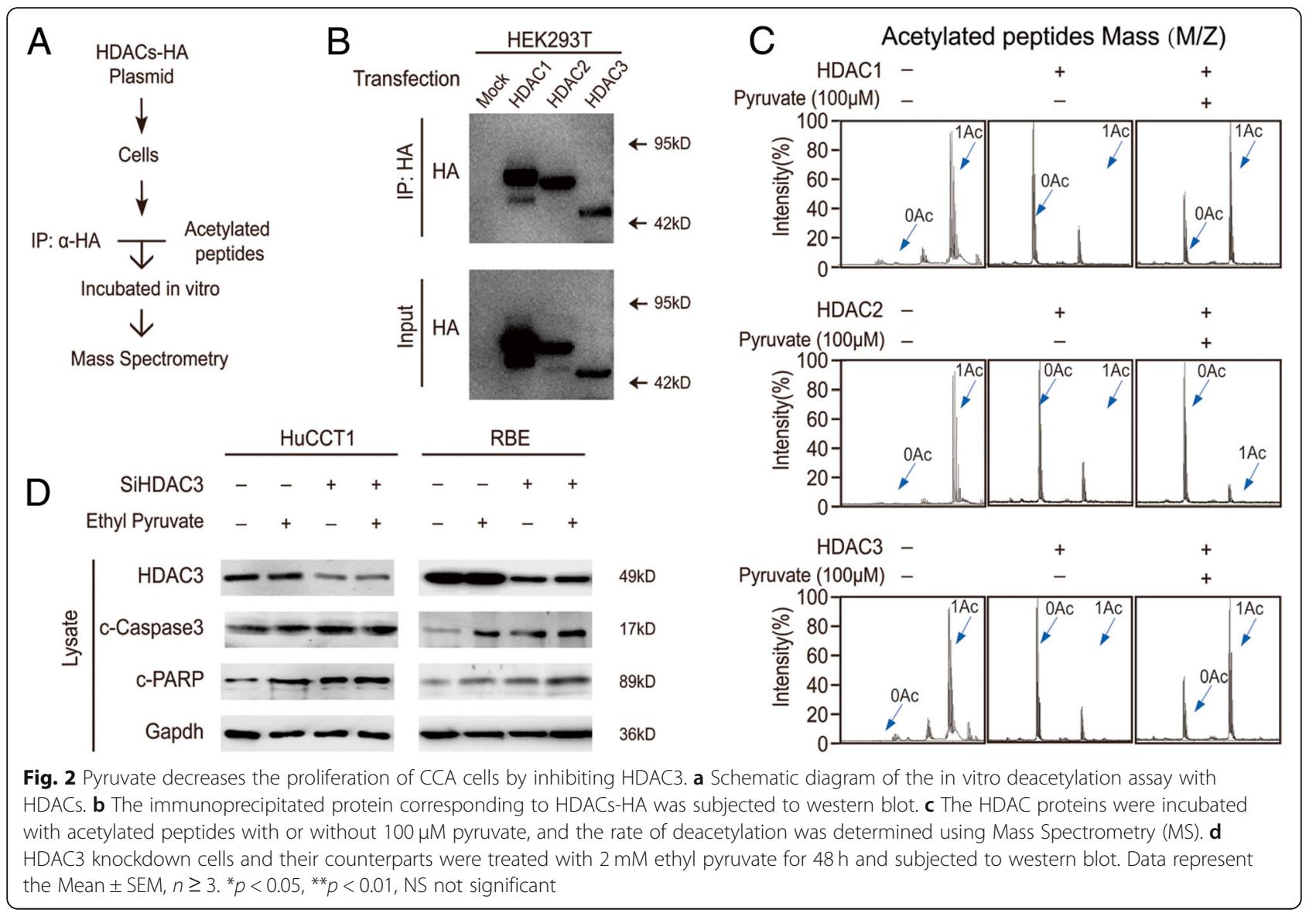

further explore whether it is possible to promote pyruvate-induced apoptosis of CCA by promoting pyruvate generation. Pyruvate is generated in tumor cells principally by glycolysis. The splice variant PKM2 is expressed specifically in cancer cells in the dimeric form with low catalytic activity, and predicts a poor prognosis in CCA patients $[2,29]$. ML265 (also called TEPP46) is a potent and selective activator of recombinant PKM2. Despite marginally increased oxygen consumption, both PKM2 activation and PKM2 overexpression did not significantly promote apoptosis of CCA cells (Fig. 3a, b $\&$ c). The dominant pyruvate consumption comes from the conversion of pyruvate into lactate. Gossypol-acetic acid (GAA) acts as an inhibitor for several dehydrogenase enzymes. This reaction is mediated by LDHA $[3,30$, 31]. We found that LDHA knockdown increased oxygen consumption, and that both LDHA inhibition and LDHA knockdown did not significantly promote apoptosis of CCA cells (Fig. 3d, e \& f). Due to the flexibility of pyruvate metabolism, targeting any of these metabolic enzymes could neither accumulate intracellular pyruvate levels nor promote apoptosis (Fig. 3a, d \& h). Finally, we promoted the formation of pyruvate and reduced the degradation of pyruvate with two drugs, and we found that apoptosis of CCA cells was significantly increased
(Fig. 3g). Accordingly, intracellular pyruvate levels were significantly increased, and oxygen consumption was extremely low, indicating that cells were on their way to apoptosis (Fig. 3g, i \& Additional file 1: Figure S1A). These results suggest that low levels of pyruvate, resulting from changes in metabolic enzymes, protected CCA cells from apoptosis.

\section{cMYC leads to a change in metabolic enzymes in CCA cells}

Tumors can change their metabolic status through differential gene expression, thereby promoting their malignant biological behavior [32]. In order to find the upstream target of changes in pyruvate metabolism, we downloaded the CCA RNA-Seq data from the TCGA database using The GDC Data Portal (https://portal.gdc.cancer.gov), which consisted of 9 normal samples and 9 paired-CCA samples. We confirmed the differentially expressed genes and then performed KEGG pathway enrichment analysis (Fig. 4a). Moreover, we observed that the most relevant pathways were metabolic and apoptosis pathways in CCA (Fig. 4b). These results suggest that the upstream gene-keeper that regulates the expression of metabolic enzymes may be principal for pyruvate metabolism in CCA. Previous studies 


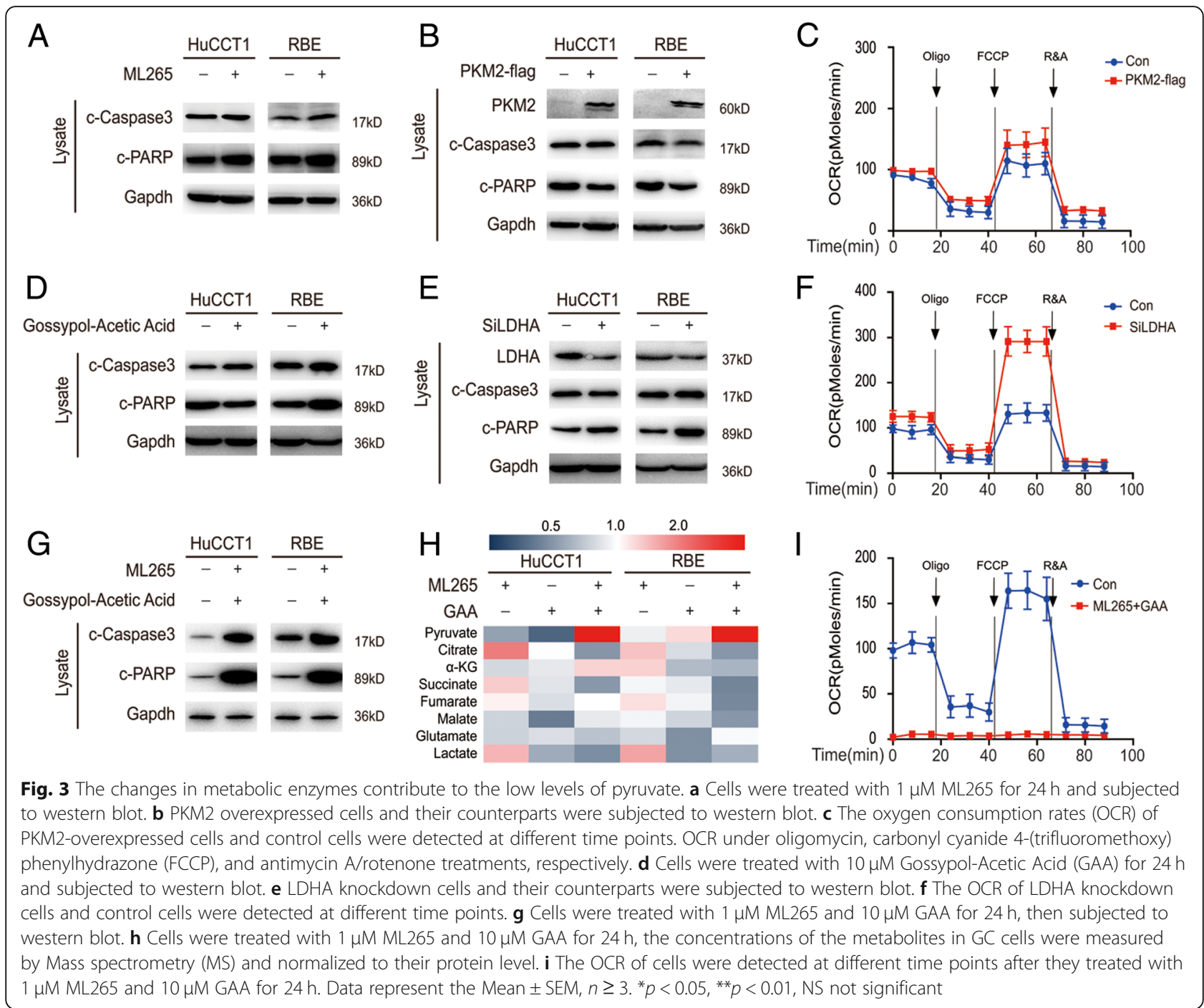

have shown that $c-M y c$, as an oncogene, has a role in contributing to tumorigenesis in many different human cancers [4, 5]. There are various mechanisms for cMYC-induced tumorigenesis, including an increased Warburg effect, and many studies have found that MYC increases metabolic proteins, such as LDHA and PKM2 $[6,7]$. Therefore, we focused on the pyruvate metabolism with cMYC. Metabolite detection demonstrated that cMYC induced a similar metabolism pattern to drug (ML265 and GAA) treatment, and this significantly increased intracellular pyruvate levels (Figs. 3k \& 4c). Pyruvate is generated in tumor cells principally by glycolysis and glutaminolysis. The levels of pyruvate in these cells are controlled not only by these two metabolic pathways, but also by the cells' ability to convert pyruvate into lactate [28]. To further identify if the pyruvate-accumulating effects of cMYC comes from glycolysis, we utilized ${ }^{13} \mathrm{C}_{6}$ glucose tracings and subsequent mass spectrometry. We found that cMYC overexpressed cells exhibited a marked increase in ${ }^{13} \mathrm{C}_{3}$-labeled lactate as well as a significant decrease in ${ }^{13} \mathrm{C}_{2}$-labeled malate. These results suggest cMYC-induced glycolysis contributed to the low pyruvate status (Fig. 4d). The expression of $c M Y C$ differs in different cancers [33,34], contributing to an abnormal metabolic status. We found cMYC knockdown simultaneously decreased the levels of PKM2 and LDHA, resulting in high intracellular pyruvate levels, as well as that cMYC overexpression could reversed it (Fig. 4c, e \& f). Moreover, the mutant type of cMYC had no effect on the levels of PKM2 and LDHA (Fig. 4g). Together, these results indicate that cMYC-induced pyruvate metabolic reprogramming is contributed to by changes of glycolytic enzymes in CCA cells.

cMYC-induced metabolic enzyme changes contribute to a poor prognosis in CCA patients

PKM2 and LDHA expression is upregulated in CCA samples, and their expression correlates with tumor 


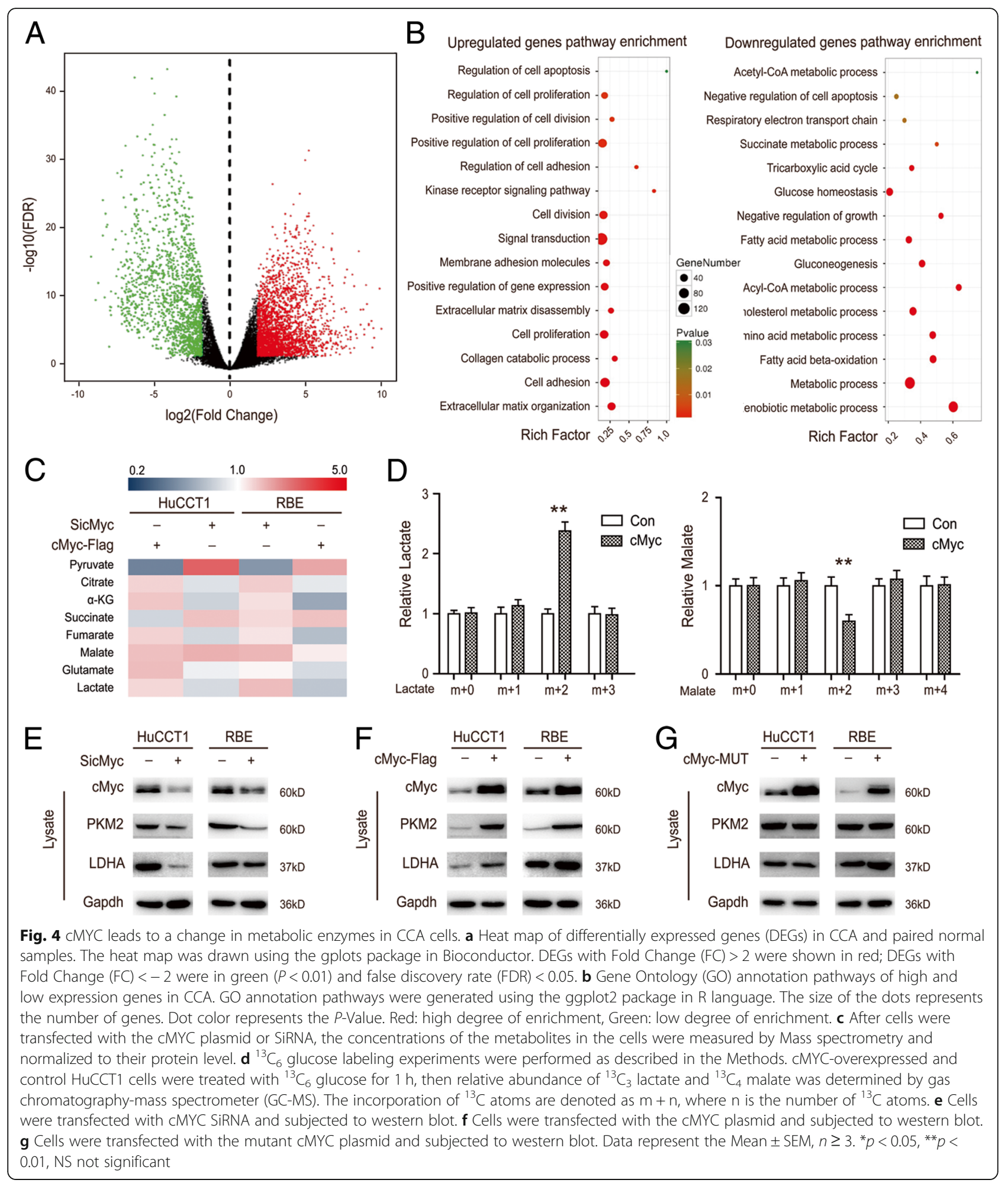

recurrence and outcome $[2,31,35]$. We used CCA tissues that contain clinically annotated data from $60 \mathrm{CCA}$ samples; the clinical characteristics of participants are summarized in Table 1. Consistent with previous studies, we found that the level of biological targets (cMYC,
PKM2 and LDHA) were increased in CCA tissues as compared to adjacent tissues (Fig. 5a \& b). Importantly, these increased biological targets were associated with a reduced patient survival (Fig. 5c). These results were further confirmed by an immunoblotting assay in fresh 


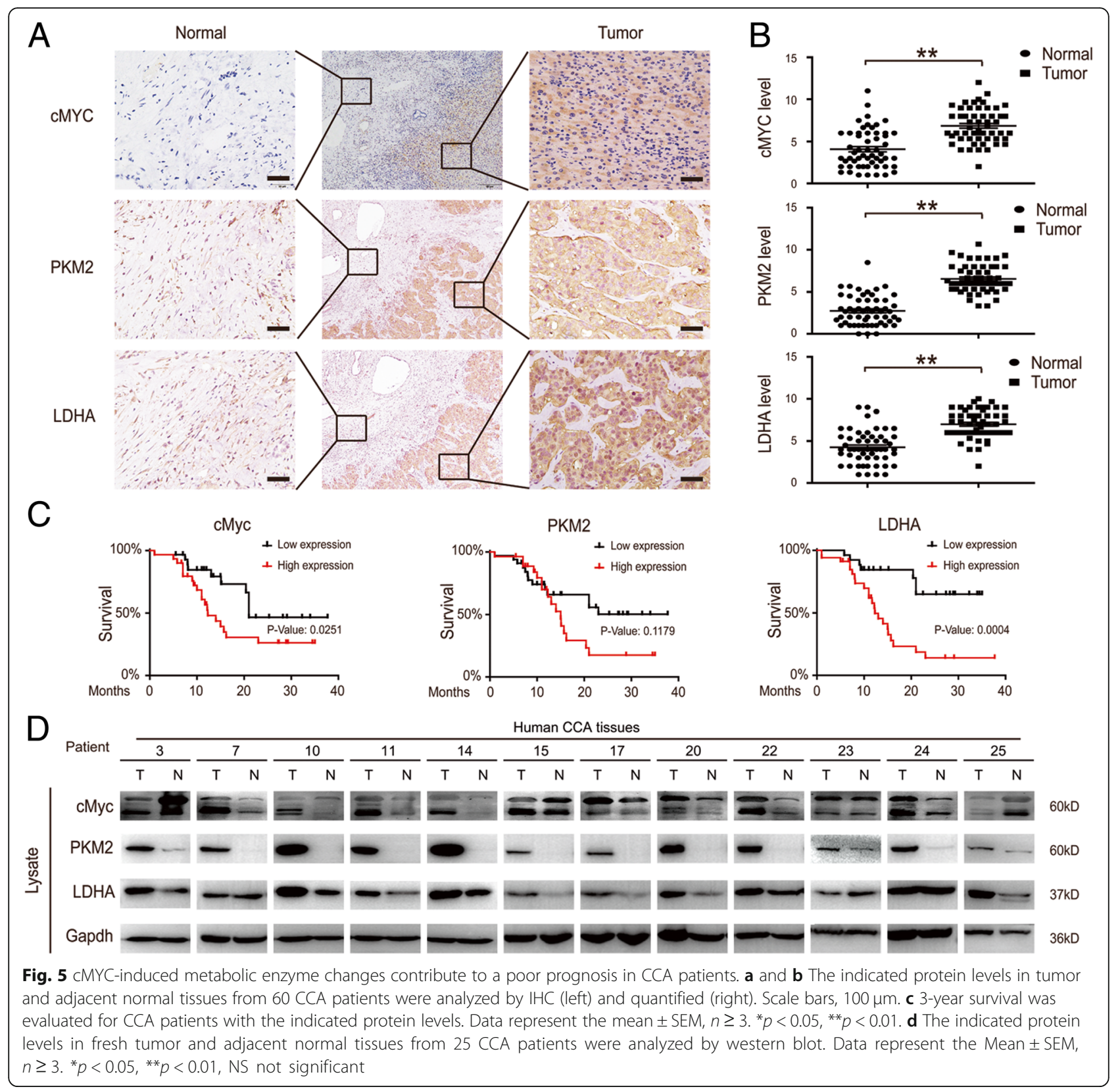

tissues (Fig. 5d). Of note, pyruvate levels in fresh tissues were negatively correlated with an increased cMYC level (Fig. 1f \& Additional file 1: Figure S1B). Collectively, these results revealed that $\mathrm{cMYC}$-induced metabolic enzyme changes contribute to a poor prognosis in CCA patients.

\section{HDAC3 deacetylates CMYC at K323 and protects CMYC from ubiquitinated degradation}

We then investigated whether the activity of HDAC3 could affect cMYC, since intracellular cMYC showed instability and is regulated by acetylation-related degradation at the K323 site in cancer cells. Moreover, HDAC3 inhibition decreases cMYC in a dose dependent fashion without altering its mRNA levels [11, 36]. We overexpressed cMYC in CCA cells, then purified the overexpressed protein by immunoprecipitation, and submitted them to both MS (mass spectrometry) as well as immunoblotting. We found that cMYC was physically intact with HDAC 3 but not the other class I HDACs (HDAC 2 shares $52 \%$ identity with HDAC3 [26, 27]) (Fig. 6a). We inhibited cMYC synthesis by using CHX (Cycloheximide) and found that $\mathrm{CMYC}$ was degraded faster in the presence of a specific HDAC3 inhibitor (RGFP966) (Fig. 6b). Furthermore, when MG132 was included to inhibit proteasomal degradation, RGFP966-induced cMYC degradation was 


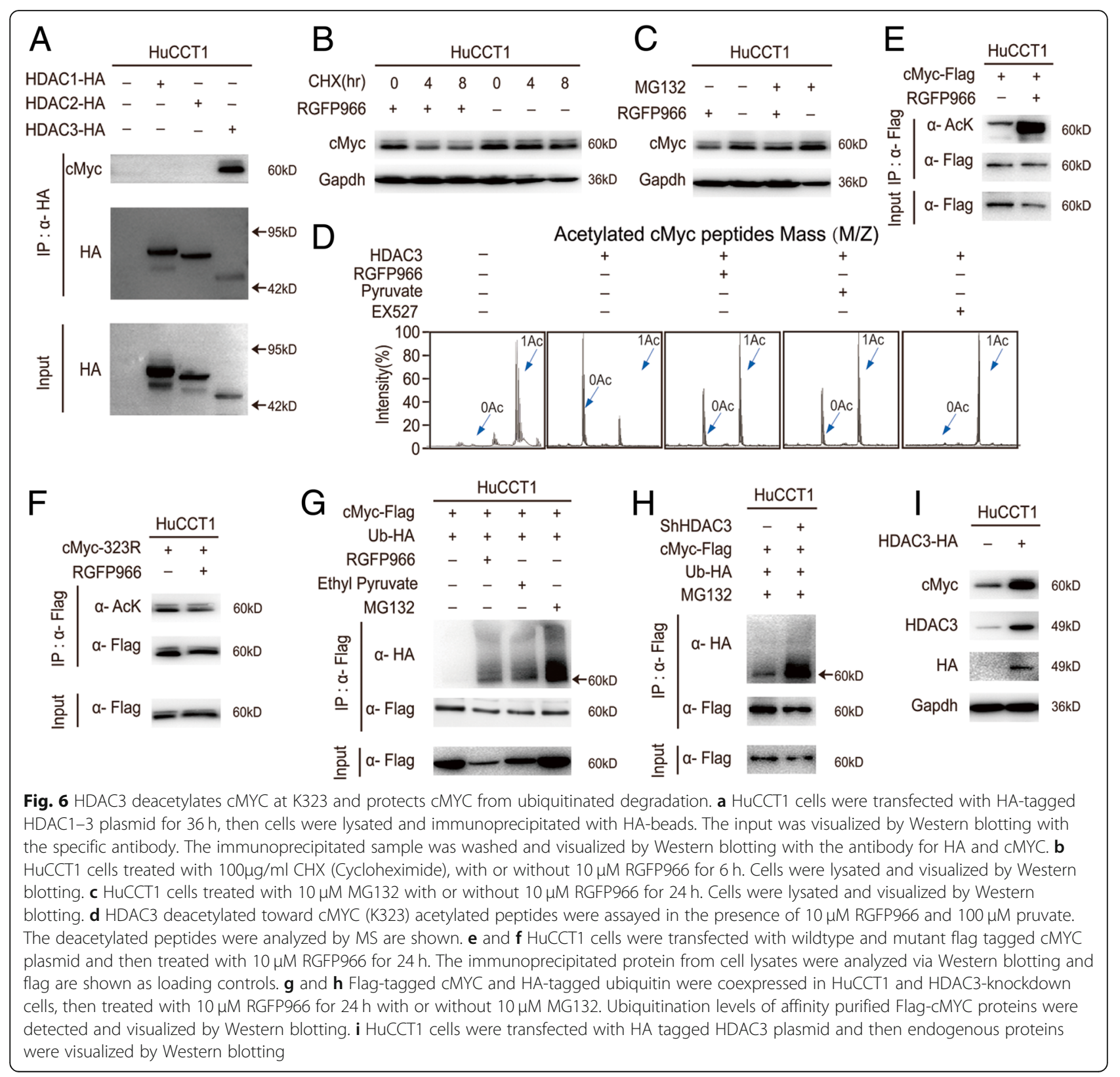

blocked (Fig. 6c). These results suggest that the ubiquitinproteasome pathway mediates an acetylation-promoted decrease of cMYC [11]. To elucidate the direct target of HDAC3, we used an in vitro deacetylation system and found that both RGFP966 and pyruvate treatment inhibited HDAC3 deacetylation at the K323 site of cMYC (Fig. 6d). Consistent with MS results, HDAC3 inhibition enhanced acetylation of wildtype cMYC but resulting in marginal acetylation change after the lysine $(\mathrm{K})$ of the K323 acetylation site was mutated to arginine (R) in CCA cells (Fig. 6e \& f). Of note, the SIRT1 inhibitor EX527 could not change the acetylation of cMYC (Fig. 6d \& Additional file 1: Figure S1C). Moreover, HDAC3 inhibition increased ubiquitination of cMYC, and conversely
HDAC3 overexpression promoted endogenous cMYC levels in CCA cells (Fig. 6g, h \& i). Together, these results suggest that HDAC3 inhibition enhanced the acetylationpromoted degradation of cMYC in CCA cells.

\section{HDAC3 inhibition induces CCA cell apoptosis by decreasing CMYC}

Previous data confirmed that the accumulated pyruvate induced by cMYC inhibition promoted apoptosis in CCA cells, so we further inquired whether promoting cMYC degradation by HDAC3 inhibition could also induce CCA apoptosis. Both RGFP966 treatment and HDAC3 knockdown significantly decreased cMYC and increased caspase substrate (polyADP ribose polymerase 
(PARP) and caspase 3) cleavage; this effect was reversed by HDAC3 overexpression (Fig. 7a, b \& Additional file 1: Figure S1D). Besides, oxygen consumption was extremely low after the treatment of RGFP966, which indicated that cells were on their way to apoptosis (Fig. 7c).
Furthermore, HDAC3 knockdown significantly inhibited xenografts (Fig. 7d) and increased the pyruvate levels as well as decreased its biological targets (cMYC) in vivo (Fig. 7e \& f). Importantly, HDAC3 knockdown induced high levels of pyruvate, which contributed to xenograft

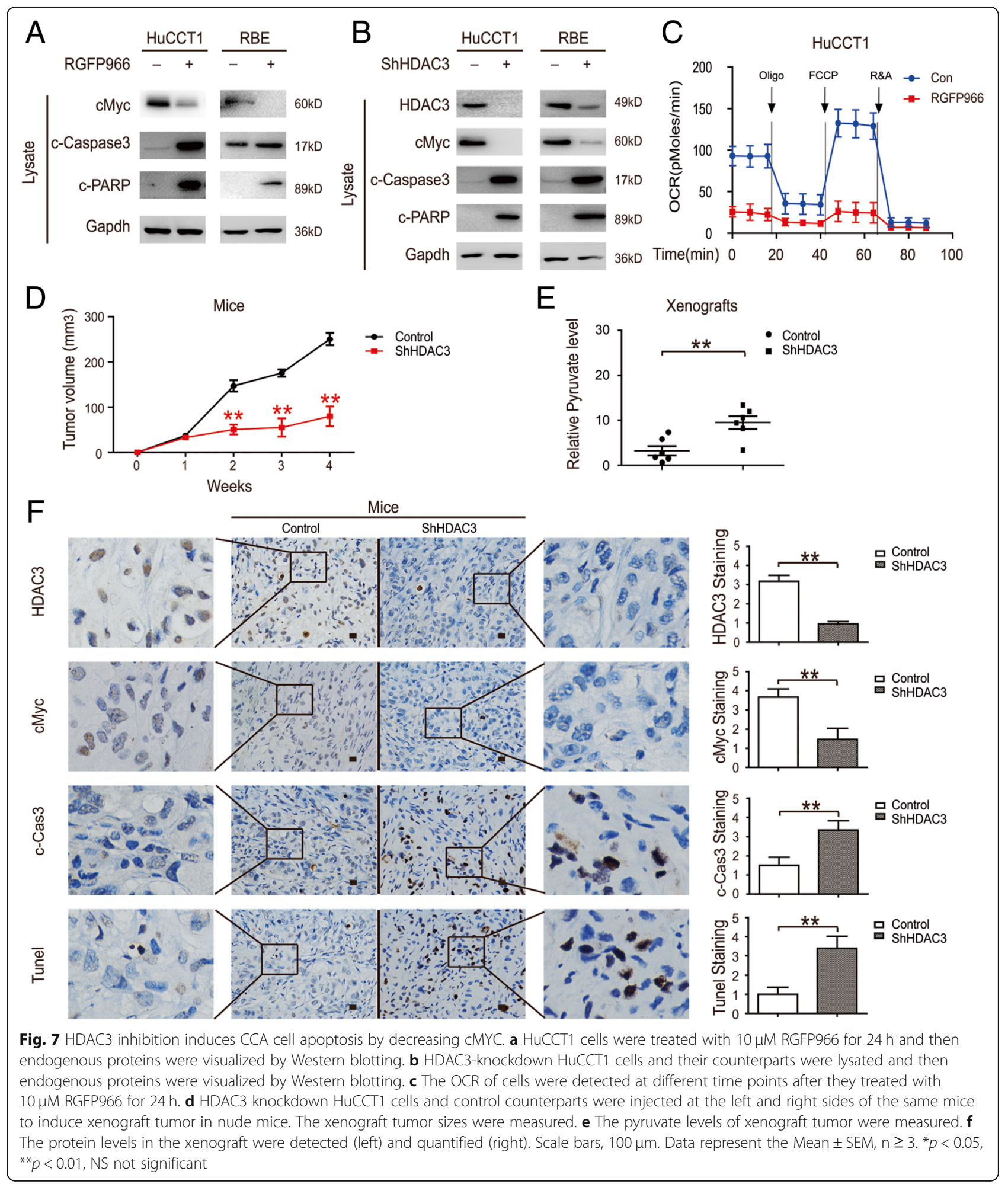


apoptosis (Fig. 7f). These results indicate that the apoptosis of CCA cells is primarily induced by HDAC3 inhibition, and cMYC-induced high levels of pyruvate may contribute to the process.

\section{Discussion}

The Warburg effect represents a serious worldwide problem threatening the health of millions of cancer patients. However, how metabolites of the Warburg effect are beneficial for its induced-tumorigenesis, and what is the downstream target of these metabolites, still remains an unanswered question. Here, we set out to identify a promoting role for the low pyruvate levels as regulated by c-Myc and its dynamic acetylation in cancer cell proliferation. Low pyruvate levels contributed via downstream targets (PKM2 and LDHA) of c-Myc and attenuated the inhibition of HDAC3 as well as decreased HDAC3-regulated apoptosis. On the contrary, a high activity of HDAC3 stabilizes the cMyc protein by preferential deacetylation of cMyc at the K323 site, which further contributes to low pyruvate levels. This creates a positive feedback loop that promotes the Warburg effect and cell proliferation of the tumor.

It is well known that the dominant metabolite of the Warburg effect is lactate, and many previous studies focus on lactate and its role in tumor proliferation [37]. Since lactate is converted from pyruvate and the end product of enhanced glycolysis is pyruvate in cancer cells, we inquired whether this decreased pyruvate benefitted tumor proliferation. The underlying notion here is that decreased generation of pyruvate in CCA is the direct result of increased glycolysis and protects the tumor from apoptosis. We demonstrated an inhibitory effect of high levels of pyruvate on CCA cells, and it is noteworthy that intracellular pyruvate levels were negatively correlated with cell viability. This may be due to the fact that the expression of SLC5A8 (the gene coding for the $\mathrm{Na}+-$ coupled pyruvate transporter that regulates the entry of blood-borne pyruvate into cancer cells) is variant in CCA cells [28]. The glycolysis induced low pyruvate levels are due to both reduced production and excessive consumption. This reduced pyruvate production comes from the expression of pyruvate kinase, the enzyme responsible for the generation of pyruvate in glycolysis. We found the splice variant PKM2 (musclespecific pyruvate kinase 2) is expressed specifically in CCA with low catalytic activity. Moreover, we confirmed the high expression of LDHA in CCA, which is effective in the conversion of pyruvate into lactate and contributed to the predominant pyruvate consumption. Collectively, the high expression of PKM2 and LDHA maintained the low intracellular levels of pyruvate in cancer cells. Interestingly, inhibition of any one of these targets could neither induce tumor proliferation, nor reverse CCA metabolic type. Yet inhibition of these three targets increased pyruvate levels while promoting apoptosis.

Since pyruvate is an energy-rich nutrient necessary for growth in non-malignant cells, there must be an upstream regulator to maintain low levels of this metabolite in cancer cells. Our findings of the present study found that the upstream regulator of pyruvate is cMYC. As an oncogene, $c-M y c$ has attracted extensive interest for its potential role in contributing to tumorigenesis. There are various mechanisms of cMYC-induced tumorigenesis, and an increased Warburg effect is one such mechanism. Many studies have also found that MYC increased metabolic proteins, such as LDHA and PKM2 [6, 7]. Thus, it has been considered a promising cancer target. Our studies demonstrate that cMYC is highly expressed in CCA and predicted a poor prognosis. Moreover, cMYC is positively correlated with PKM2 and LDHA, and contributed to the low pyruvate level. The stability of c-Myc protein is related to its acetylation at K323 [9, 10], and HDACi treatment, but not SIRTi treatment, induced c-Myc K323 acetylation as well as tumorigenesis inhibition [11, 12]. Employing immunoprecipitation, we further inquired as to which HDAC interacted with $\mathrm{CMYC}$, and found that HDAC3 deacetylated cMYC at K323 and further protected cMYC from ubiquitinated degradation. Our work establishes cMYC inhibition as a strategy to accumulate pyruvate, which is effective in promoting apoptosis in CCA cells.

After confirming that the upstream regulator of pyruvate is cMYC, we moved to the other side and inquired as to what is the downstream target of pyruvate in CCA. Previous studies have concluded that the tumor suppressive function of pyruvate is related to its ability to inhibit HDACs; pyruvate is an HDAC inhibitor and a tumor suppressor [28]. Here we confirmed HDAC3 is the downstream target of pyruvate. It is important to note that HDAC3, which is capable of being inhibited by pyruvate, is up-regulated in CCA and protects CCA cells form apoptosis [18]. The elevation of HDAC3 activity is presumably necessary for the cancer cells to maintain their malignant phenotype [28]. Therefore, CCA cells must maintain low intracellular levels of pyruvate, lest HDAC3 will be inhibited and cell growth prevented by enhanced apoptosis.

Deregulated apoptosis by histone acetylation is one of the mechanisms related to tumorigenesis [20]. Histone deacetylases (HDACs) catalyze the removal of acetyl groups from lysine tails. Since HDACs inhibit specific tumor suppressor genes, resulting in an aberrant epigenetic status and low apoptosis level of cancer cells, they may serve as candidate anti-cancer targets [38, 39]. Moreover, we have demonstrated that increased expression and activation of HDAC3 plays an anti-apoptotic role in epigenetic alterations, impacting pro-apoptotic 


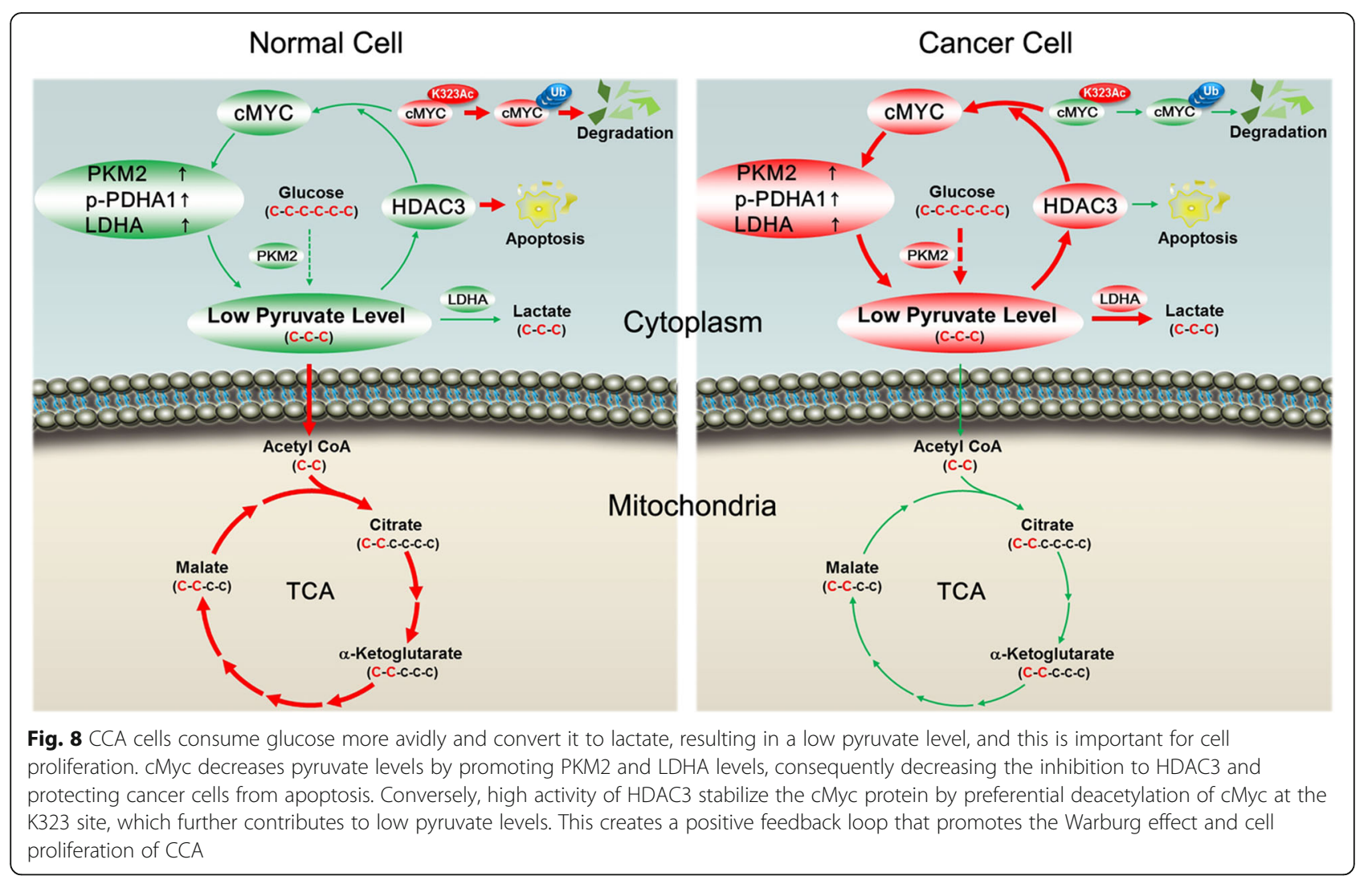

pathways in CCA but not in normal cells [18]. Here, we demonstrated that cMYC-induced low intracellular levels of pyruvate decreased HDAC3 inhibition and promoted CCA proliferation. Interestingly, $\mathrm{cMyc}$ and HDAC3 constitutes a positive feedback loop, which is connected by pyruvate: cMyc decreases pyruvate levels by promoting PKM2 and LDHA levels, consequently decreasing inhibition to HDAC3 and protecting cancer cells from apoptosis. Conversely, high activity of HDAC3 stabilizes cMyc protein by preferential deacetylation of cMyc at the K323 site, which further contributes to the low pyruvate levels. This creates a positive feedback loop that promotes the Warburg effect and cell proliferation of the tumor (Fig. 8). To our knowledge, this is the first report describing this unique phenomenon of the positive feedback loop in cancer cells.

\section{Conclusions}

In the present study, we set out to identify a promoting role for low pyruvate levels regulated by c-Myc and its dynamic acetylation in cancer cell proliferation. Moreover, we reported a unique phenomenon of the positive feedback loop with cMyc and HDAC3. Therapeutic strategies targeting pyruvate may be applicable to cancer treatment in a wide variety of tissues, since high expression of c-Myc is a common phenomenon in cancer.

\section{Additional file}

Additional file 1: Low pyruvate levels protects cholangiocarcinoma. (A) The OCR of cells were detected at different time points after they treated with pyruvate and ethyl pyruvate for 48h. (B) CMYC protein levels in fresh tumor tissues from 25 CCA patients were analyzed by western blot. Then the correlation between the CMYC levels and their pyruvate levels were analysed and normalized to the protein level. C) HuCCT1 cells were transfected with flag tagged CMYC plasmid and then treated with $100 \mathrm{nM}$ EX527 for 48 hours. The immunoprecipitated protein from cell lysates were analyzed via Western blotting and flag are shown as loading controls. (D) HDAC3-overexpressed cells and their counterparts were lysated, and then endogenous proteins were visualized by Western blotting. (TIF $1437 \mathrm{~kb}$ )

\section{Abbreviations}

CCA: Cholangiocarcinoma; CHX: Cycloheximide; HDAC: Histone deacetylase: HDACi: HDAC inhibitiors; LDH: Lactate Dehydrogenase; MS: mass spectrometry; PARP: polyADP ribose polymerase; PKM2: Muscle specific Pyruvate Kinase 2; SIRT: sirtuins

\section{Acknowledgements}

We thank the Zhao lab for offering their help.

\section{Funding}

This work was supported by grants from the National Natural Science Foundation of China (No. 81602076, 81672935 and 81472756), Outstanding Youth Project of Nanjing City (No. JQX17002), the Jiangsu Clinical Medical Center of Digestive Disease (BL2012001), the Natural Science Foundation from the Department of Science \& Technology of Jiangsu Province (BK20160113), the Fund of Jiangsu Provincial Commission of Health and Family Planning (No. Q201611), the Fundamental Research Funds for the Central Universities (No. 021414380244) and Macau Science and Technology Development Fund (009/2017/A1). 


\section{Availability of data and materials}

All data generated or analysed during this study are included in this published article [and its supplementary information files].

\section{Authors' contributions}

$M Z$, LW and $X Z$ designed the study; QZ, DT, YL and YP did the cell experiments; YL, YY, YW and LX collected the tissue samples; $Q Z$ and $Y L$ performed the protein analysis; $L Z, Y W, M Z$ and $Y L$ drafted the manuscript and performed the immunohistochemistry experiment; BK, LW and JW performed the metabolite analysis; RGD and MZ wrote the manuscript, $M Z$, HF, SZ and XZ supported the study. All authors read and approved the fina manuscript.

\section{Ethics approval and consent to participate}

All experiments utilizing animal and human samples were approved by the Ethical Committee of Medical Research, Nanjing Drum Tower Hospital, Affiliated Hospital of Nanjing University Medical School.

\section{Consent for publication}

$$
\text { Not applicable }
$$

\section{Competing interests}

The authors declare that they have no competing interests.

\section{Publisher's Note}

Springer Nature remains neutral with regard to jurisdictional claims in published maps and institutional affiliations.

\begin{abstract}
Author details
${ }^{1}$ Department of Gastroenterology, Nanjing Drum Tower Hospital, the Affiliated Hospital of Nanjing University Medical School, Nanjing University, No.321 Zhongshan Road, 210008 Nanjing, People's Republic of China. ${ }^{2}$ Key laboratory of Reproduction Regulation of NPFPC (SIPPR, IRD), Fudan University, Shanghai 200032, China. ${ }^{3}$ Department of Digestive Diseases of Huashan Hospital, Shanghai, China. ${ }^{4}$ School of Life Sciences, Fudan University, Shanghai, China. ${ }^{5}$ Northwestern University Feinberg School of Medicine, Chicago, IL, USA. 'Department of Gastroenterology, Nanjing Medical University Affiliated Drum Tower Clinical Medical College, Nanjing Medical University, Nanjing, China. ${ }^{7}$ Department of Surgery, Technical University of Munich (TUM), Munich, Germany. ${ }^{8}$ State Key Laboratory of Quality Research in Chinese Medicine, Macau Institute for Applied Research in Medicine and Health, Faculty of Chinese Medicine, Macau University of Science and Technology, Avenida Wai Long, Taipa, Macao 442000, People's Republic of China.
\end{abstract}

Received: 15 October 2018 Accepted: 20 February 2019 Published online: 12 March 2019

\section{References}

1. Warburg O. On the origin of cancer cells. Science. 1956;123(3191):309-14.

2. $Y u$ G, et al. PKM2 regulates neural invasion of and predicts poor prognosis for human hilar cholangiocarcinoma. Mol Cancer. 2015;14:193.

3. Kawamoto M. Breast cancer diagnosis by lactate dehydrogenase isozymes in nipple discharge. Cancer. 1994;73(7):1836-41.

4. Meyer N, Penn LZ. Reflecting on 25 years with MYC. Nat Rev Cancer. 2008; 8(12):976-90.

5. Jing $H$, et al. A SIRT2-selective inhibitor promotes c-Myc Oncoprotein degradation and exhibits broad anticancer activity. Cancer Cell. 2016; 29(5):767-8.

6. Kim JW, et al. Evaluation of myc E-box phylogenetic footprints in glycolytic genes by chromatin immunoprecipitation assays. Mol Cell Biol. 2004;24(13): 5923-36.

7. David CJ, et al. HnRNP proteins controlled by c-Myc deregulate pyruvate kinase mRNA splicing in cancer. Nature. 2010;463(7279):364-8.

8. Delmore JE, et al. BET bromodomain inhibition as a therapeutic strategy to target c-Myc. Cell. 2011;146(6):904-17.

9. Patel $\mathrm{JH}$, et al. The c-MYC oncoprotein is a substrate of the acetyltransferases hGCN5/PCAF and TIP60. Mol Cell Biol. 2004;24(24):10826-34.

10. Faiola F, et al. Dual regulation of c-Myc by p300 via acetylation-dependent control of Myc protein turnover and coactivation of Myc-induced transcription. Mol Cell Biol. 2005;25(23):10220-34.
11. Nebbioso A, et al. C-Myc modulation and acetylation is a key HDAC inhibitor target in Cancer. Clin Cancer Res. 2017;23(10):2542-55.

12. Simmons JK, et al. Cooperative targets of combined mTOR/HDAC inhibition promote MYC degradation. Mol Cancer Ther. 2017;16(9):2008-21.

13. Lakshmaiah KC, et al. Epigenetic therapy of cancer with histone deacetylase inhibitors. J Cancer Res Ther. 2014;10(3):469-78.

14. Fullgrabe J, Kavanagh E, Joseph B. Histone onco-modifications. Oncogene. 2011;30(31):3391-403.

15. Zhao S, et al. Regulation of cellular metabolism by protein lysine acetylation. Science. 2010;327(5968):1000-4.

16. Song SH, Han SW, Bang YJ. Epigenetic-based therapies in cancer: progress to date. Drugs. 2011;71(18):2391-403.

17. Arts J, et al. JNJ-26481585, a novel "second-generation" oral histone deacetylase inhibitor, shows broad-spectrum preclinical antitumoral activity. Clin Cancer Res. 2009;15(22):6841-51.

18. Yin $Y$, et al. Histone deacetylase 3 overexpression in human cholangiocarcinoma and promotion of cell growth via apoptosis inhibition. Cell Death Dis. 2017;8(6):e2856.

19. Zhang $M$, et al. AR-42 induces apoptosis in human hepatocellular carcinoma cells via HDAC5 inhibition. Oncotarget. 2016;7(16):22285-94.

20. Zhijun $\mathrm{H}$, et al. Pre-clinical characterization of 4SC-202, a novel class I HDAC inhibitor, against colorectal cancer cells. Tumour Biol. 2016;37(8):10257-67.

21. Richon VM, et al. A class of hybrid polar inducers of transformed cell differentiation inhibits histone deacetylases. Proc Natl Acad Sci U S A. 1998; 95(6):3003-7.

22. Minami J, et al. Histone deacetylase 3 as a novel therapeutic target in multiple myeloma. Leukemia. 2014;28(3):680-9.

23. Frezza $C$, et al. Haem oxygenase is synthetically lethal with the tumour suppressor fumarate hydratase. Nature. 2011;477(7363):225-8.

24. Xiao Y, et al. Ku80 cooperates with CBP to promote COX-2 expression and tumor growth. Oncotarget. 2015;6(10):8046-61.

25. Thangaraju M, et al. SLC5A8 triggers tumor cell apoptosis through pyruvatedependent inhibition of histone deacetylases. Cancer Res. 2006;66(24):11560-4.

26. Hagelkruys A, et al. The biology of HDAC in cancer: the nuclear and epigenetic components. Handb Exp Pharmacol. 2011;206:13-37.

27. Yang WM, et al. Isolation and characterization of cDNAs corresponding to an additional member of the human histone deacetylase gene family. J Biol Chem. 1997;272(44):28001-7.

28. Thangaraju $\mathrm{M}$, et al. Colon cancer cells maintain low levels of pyruvate to avoid cell death caused by inhibition of HDAC1/HDAC3. Biochem J. 2009; 417(1):379-89.

29. Mazurek $\mathrm{S}$, et al. Pyruvate kinase type $\mathrm{M} 2$ and its role in tumor growth and spreading. Semin Cancer Biol. 2005;15(4):300-8.

30. Singh $R$, et al. Lactate dehydrogenase $(\mathrm{LDH})$ isoenzymes patterns in ocular tumours. Indian J Ophthalmol. 1991;39(2):44-7.

31. Yu Y, et al. Overexpression of lactate dehydrogenase-a in human intrahepatic cholangiocarcinoma: its implication for treatment. World J Surg Oncol. 2014:12:78.

32. Shibata T, Aburatani H. Exploration of liver cancer genomes. Nat Rev Gastroenterol Hepatol. 2014;11(6):340-9.

33. Chen J, et al. SIRT2 overexpression in hepatocellular carcinoma mediates epithelial to mesenchymal transition by protein kinase B/glycogen synthase

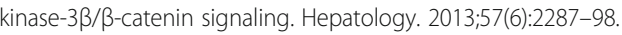

34. Bajpe PK, et al. A chromatin modifier genetic screen identifies SIRT2 as a modulator of response to targeted therapies through the regulation of MEK kinase activity. Oncogene. 2015;34(4):531-6.

35. Thonsri $U$, et al. Overexpression of lactate dehydrogenase a in cholangiocarcinoma is correlated with poor prognosis. Histol Histopathol. 2017;32(5):503-10.

36. Harada T, et al. HDAC3 regulates DNMT1 expression in multiple myeloma: therapeutic implications. Leukemia. 2017:31(12):2670-77.

37. Zhao D, et al. Lysine-5 acetylation negatively regulates lactate dehydrogenase a and is decreased in pancreatic cancer. Cancer Cell. 2013;23(4):464-76.

38. Witt $\mathrm{O}$, et al. HDAC family: what are the cancer relevant targets? Cancer Lett. 2009;277(1):8-21.

39. Mariadason JM. HDACs and HDAC inhibitors in colon cancer. Epigenetics. 2008;3(1):28-37. 